\title{
HIGH-RESOLUTION INFRARED IMAGING AND SPECTROSCOPY OF THE Z CANIS MAJORIS SYSTEM DURING QUIESCENCE AND OUTBURST
}

\author{
Sasha Hinkley ${ }^{1,13}$, Lynne Hillendrand ${ }^{1}$, Ben R. Oppenheimer ${ }^{2}$, Emily L. Rice ${ }^{3,2}$, Laurent Pueyo ${ }^{4,14}$, Gautam Vasisht ${ }^{5}$, \\ Neil Zimmerman $^{2,6}$, Adam L. Kraus ${ }^{7}$, Michael J. Ireland ${ }^{8,9}$, Douglas Brenner ${ }^{2}$, Charles Beichman ${ }^{10}$, \\ Richard Dekany ${ }^{11}$, Jennifer E. Roberts ${ }^{5}$, Ian R. Parry ${ }^{12}$, Lewis C. Roberts JR. ${ }^{5}$, Justin R. CrepP ${ }^{1,15}$, \\ Rick Burruss ${ }^{5}$, J. Kent Wallace ${ }^{5}$, Eric Cady $^{5}$, ChengXing Zhai ${ }^{5}$, Michael ShaO ${ }^{5}$, Thomas Lockhart ${ }^{5}$, \\ RÉmi SOUMmer ${ }^{4}$, AND ANAND SivaramaKRISHNAN ${ }^{4}$ \\ ${ }^{1}$ Department of Astronomy, California Institute of Technology, 1200 E. California Blvd, MC 249-17, Pasadena, CA 91125, USA \\ 2 Astrophysics Department, American Museum of Natural History, Central Park West at 79th Street, New York, NY 10024, USA \\ ${ }^{3}$ Department of Engineering Science and Physics, College of Staten Island, City University of New York, \\ 2800 Victory Bvld, Staten Island, NY 10314, USA \\ ${ }^{4}$ Space Telescope Science Institute, 3700 San Martin Drive, Baltimore, MD 21218, USA \\ 5 Jet Propulsion Laboratory, California Institute of Technology, 4800 Oak Grove Dr., Pasadena, CA 91109, USA \\ ${ }^{6}$ Max Planck Institute for Astronomy, Königstuhl 17, D-69117 Heidelberg, Germany \\ ${ }^{7}$ Harvard-Smithsonian Center for Astrophysics, 60 Garden Street, Cambridge, MA, 02138, USA \\ ${ }^{8}$ Department of Physics and Astronomy, Macquarie University, New South Wales, NSW 2109, Australia \\ ${ }_{9}$ Australian Astronomical Observatory, P. O. Box 296, Epping, NSW 1710, Australia \\ ${ }^{10}$ NASA Exoplanet Science Institute, California Institute of Technology, Pasadena, CA 91125, USA \\ ${ }^{11}$ Caltech Optical Observatories, California Institute of Technology, Pasadena, CA 91125, USA \\ ${ }^{12}$ Institute of Astronomy, University of Cambridge, Madingley Road, Cambridge CB3 0HA, UK \\ Received 2012 August 2; accepted 2012 December 4; published 2012 December 28
}

\begin{abstract}
We present adaptive optics photometry and spectra in the $J H K L$ bands along with high spectral resolution $K$-band spectroscopy for each component of the Z Canis Majoris system. Our high angular resolution photometry of this very young $(\lesssim 1 \mathrm{Myr}$ ) binary, comprised of an FU Ori object and a Herbig Ae/Be star, was gathered shortly after the 2008 outburst while our high-resolution spectroscopy was gathered during a quiescent phase. Our photometry conclusively determines that the outburst was due solely to the embedded Herbig Ae/Be member, supporting results from earlier works, and that the optically visible FU Ori component decreased slightly $(\sim 30 \%)$ in luminosity during the same period, consistent with previous works on the variability of FU Ori type systems. Further, our high-resolution $K$-band spectra definitively demonstrate that the $2.294 \mu \mathrm{m} \mathrm{CO}$ absorption feature seen in composite spectra of the system is due solely to the FU Ori component, while a prominent CO emission feature at the same wavelength, long suspected to be associated with the innermost regions of a circumstellar accretion disk, can be assigned to the Herbig Ae/Be member. These findings clarify previous analyses of the origin of the $\mathrm{CO}$ emission in this complex system.
\end{abstract}

Key words: binaries: close - instrumentation: adaptive optics - stars: individual (Z CMa) - stars: pre-main sequence

Online-only material: color figures

\section{INTRODUCTION}

Z Canis Majoris (hereafter " $\mathrm{Z}$ CMa") was one of the original members of the set of $\mathrm{Ae}$ and $\mathrm{Be}$ stars with nebulosity first reported in Herbig (1960), now called the Herbig Ae/Be type stars. This classification alludes not only to its early spectral type and emission lines, but also to brightness fluctuations and heavy nebulosity. At a distance of 1150 pc (Clariá 1974), Z CMa is part of the CMa T1 association with a quoted age $<1$ Myr (Herbst et al. 1978). Consistent with its classification and presumed age, the Z CMa system shows strong P Cyg profiles in lines of $\mathrm{H} \alpha, \mathrm{H} \beta$, and Fe II (Covino et al. 1984), radio continuum emission, bipolar jets as well as strong infrared excess (Poetzel et al. 1989). Initial efforts to image the emitting source at high resolution revealed a $\sim 100$ mas elongation (Leinert \& Haas 1987), suggesting a nearly edge-on disk-like structure. Shortly

\footnotetext{
${ }^{13}$ NSF Fellow.

${ }^{14}$ Sagan Fellow.

${ }^{15}$ Current address: Department of Physics, University of Notre Dame, 225

Nieuwland Science Hall, Notre Dame, IN 46556, USA.
}

thereafter, Koresko et al. (1989) suggested that the extended emission was probably not due to a disk, but rather a cooler, close companion retaining luminosity from its pre-main-sequence contraction.

At about the same time, Hartmann et al. (1989) suggested that the Z CMa system had many characteristics of FU-Orilike systems (see, e.g., Hartmann \& Kenyon 1996), including a bright accretion disk with a high rate of accretion, and signatures of powerful winds. Progress on reconciling the Herbig $\mathrm{Ae} / \mathrm{Be}$ nature of the star with its now apparent FU-Ori-like characteristics was achieved when evidence was uncovered through speckle imaging for two objects comprising the system (Koresko et al. 1991). These measurements were later verified by visible direct imaging (Thiebaut et al. 1995), revealing two clearly defined point sources. Such results provided support to the hypothesis that a significant fraction of FU Ori type systems may be binary systems (Bonnell \& Bastien 1992), which is indeed the case for the FU Ori system itself (Wang et al. 2004; Reipurth \& Aspin 2004, Pueyo et al. 2012b).

Synthesizing several decades of observations, a consistent picture of the $\mathrm{Z} \mathrm{CMa}$ system has now emerged of a binary 
Table 1

Table of Observations

\begin{tabular}{|c|c|c|c|c|c|c|}
\hline Object & Star Type & Date & Z CMa State & Observatory and Instrument & $\begin{array}{l}\text { Wavelengths } \\
\qquad(\mu \mathrm{m})\end{array}$ & Mode \\
\hline $\mathrm{Z} \mathrm{CMa}$ & Target & 2006 Dec 17 & Quiescence & Keck: NIRSPEC & $2.10-2.13,2.29-2.32$ & Spectroscopy \\
\hline S Mon & Calibrator & 2006 Dec 17 & $\ldots$ & Keck: NIRSPEC & $2.10-2.13,2.29-2.32$ & Spectroscopy \\
\hline $\mathrm{Z} \mathrm{CMa}$ & Target & 2008 Dec 21 & Outburst & Keck: NIRC2 & $1.57-3.32$ & Imaging \\
\hline HD 53455 & Calibrator & 2008 Dec 21 & $\ldots$ & Keck: NIRC2 & $1.57-3.32$ & Imaging \\
\hline HD 75898 & Calibrator & 2008 Dec 21 & $\ldots$ & Keck: NIRC2 & $1.57-3.32$ & Imaging \\
\hline HD 112196 & Calibrator & 2009 Mar 17 & $\ldots$ & Palomar: Project 1640 & $1.06-1.76$ & Spectrophotometry \\
\hline
\end{tabular}

system comprised of a FU-Ori-like object which dominates the optical emission and a Herbig Ae/Be object which dominates the infrared (Whitney et al. 1993; van den Ancker et al. 2004). The terminology used for each component in the system in past literature has been somewhat ambiguous given the constantly evolving physical understanding of this remarkable system. As such, in this work we have chosen to abandon the "primary" and "secondary" nomenclature that pervades the literature, and instead use the terms "Z CMa Southeast (SE)" to refer to the FU Ori component and "Z CMa Northwest (NW)," referring to Herbig Ae/Be component. Koresko et al. (1991) show that the SE component dominates the emission of the NW component blueward of $2 \mu \mathrm{m}$, and vice versa for wavelengths longer than $2 \mu \mathrm{m}$.

Support for the basic physical picture of the system has been verified repeatedly over the past 10 years, i.e., the identification that the SE component is a $\sim$ few $M_{\odot}$ object (van den Ancker et al. 2004) responsible for the jet/outflow phenomena (Millan-Gabet \& Monnier 2002), while the NW component has considerable evidence supporting its Herbig Ae/Be nature including emission lines and a compact, massive envelope (Whitney et al. 1993). This system experienced a significant outburst in 2008 (e.g., Grankin \& Artemenko 2009) lasting $\sim 1.5$ years, and was photometrically monitored extensively at X-ray to visible wavelengths (Malbet et al. 2010). Indeed, using spectropolarimetry of the system, Szeifert et al. (2010) claimed the outburst was associated with the Herbig $\mathrm{Ae} / \mathrm{Be}$ component of the system. Further, while dual-imaging polarimetry has typically been reserved for young circumstellar disk systems (e.g., Oppenheimer et al. 2008; Hinkley et al. 2009), recently Canovas et al. (2012) have used this technique to identify the jets associated with each member of this system.

Near-infrared spectroscopy of the Z CMa system has been scarce over the past 30 years. Indeed, as Antoniucci et al. (2009) point out, only Liljestroem \& Olofsson (1997) and van den Ancker et al. (2004) have provided previous nearinfrared spectra. In this Letter, we present near-IR spectra and/or photometry in the JHKL bands for both components during the 2008 outburst, resolved using high angular resolution imaging (Oppenheimer \& Hinkley 2009), as well as archival photometry covering the same bandpasses (Koresko et al. 1991) for comparison. Our adaptive optics ("AO”) measurements directly demonstrate and provide confirmation of previous work (e.g., Szeifert et al. 2010; Malbet et al. 2010) that suggested the outburst is due to the Herbig Ae/Be component, although the exact mechanism of the brightening of this member remains unknown. Moreover, we present high-resolution $K$-band spectra of each member of the binary obtained in 2006 during the quiescent phase. Table 1 provides a list of our observations.

\section{OBSERVATIONS AND DATA PROCESSING}

Our high angular resolution observations of $\mathrm{Z}$ CMa were taken using Palomar/P1640 and Keck/NIRC2 AO during outburst. Our AO-assisted $K$-band spectroscopy was taken with Keck/NIRSPEC in quiescence. Table 1 provides a summary.

\subsection{JHKL-band Spectra and Photometry During Outburst}

We imaged the $\mathrm{Z}$ CMa system in its high state on UT 2009 March 17 using "Project 1640" (Hinkley et al. 2011c; Oppenheimer et al. 2012) on the 200 inch Hale Telescope at Palomar Observatory. Project 1640 is a coronagraph integrated with an integral field spectrograph (IFS). The IFS+Coronagraph package is mounted on the Palomar AO system (Dekany et al. 1998), which in turn is mounted at the Cassegrain focus of the Hale Telescope. The coronagraph is an Apodized-Pupil Lyot coronagraph (Sivaramakrishnan et al. 2001; Soummer 2005), having a 370 mas diameter $(5.37 \lambda / D$ at $H$ band) focal plane mask. The IFS is a microlens-based imaging spectrograph which can simultaneously obtain $\sim 40,000$ spectra across our 3". $8 \times 3$ 3. 8 field of view. Each microlens subtends 19.2 mas on the sky and a dispersing prism provides a spectral resolution $(\lambda / \Delta \lambda) \sim 45$. The observing wavelengths spanned the $J$ and $H$ bands (1.06-1.76 $\mu \mathrm{m}$ at the time of these observations). Early examples of spectrophotometry and astrometry from this project can be found in Hinkley et al. (2010, 2011b), Zimmerman et al. (2010), Roberts et al. (2012), and Pueyo et al. (2012b). While an IFS clearly aids in spectral characterization, it can also improve sensitivity (Crepp et al. 2011; Pueyo et al. 2012a) through the suppression of quasi-static speckle noise, which will limit high contrast observations (Hinkley et al. 2007). This system is also equipped with aperture masking interferometry capabilities (e.g., Hinkley et al. 2011a; Kraus \& Ireland 2012) for the characterization of systems with very small angular separations, although this technique was not employed in this study.

Three individual wavelength channels are shown in Figure 1. To fully resolve both members of the system, the target was observed $\sim 1^{\prime \prime}$ away from the coronagraphic mask. Pointspread function (PSF) fitting photometry was performed in each of the 23 wavelength channels using a calibration star, HD 112196 (F8V, $V=7.01)$, obtained on the same night with similar signal-to-noise and observed at similar airmass. The 100 mas angular separation of the two Z CMa components (Figure 1) is only slightly larger than the 70 mas $H$-band diffraction limit of the Palomar Hale Telescope. Given this fact, fitting the empirical calibration PSF star to each member was performed simultaneously. Each channel of the Z CMa images was oversampled by a factor of five, and the calibration PSF was fit to each Z CMa component to determine peak brightness and centroid positions. Once these two best-fit 


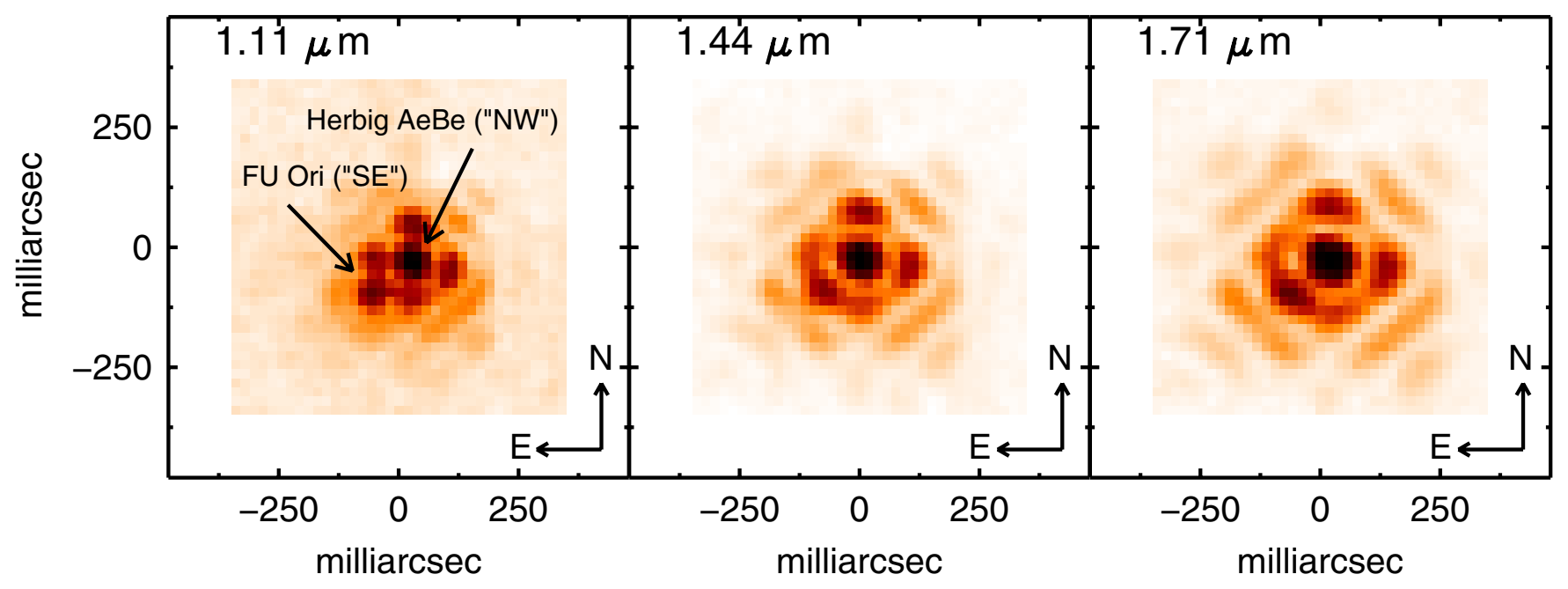

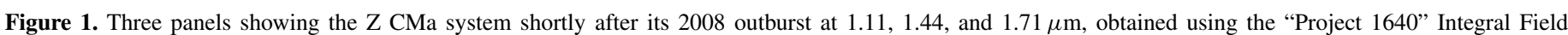

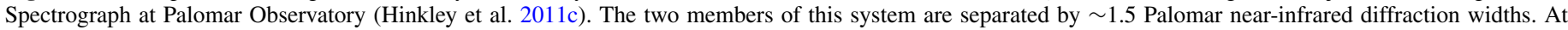

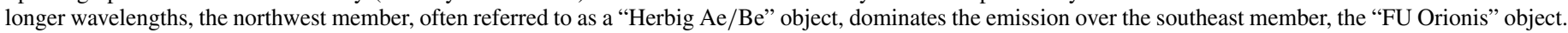
(A color version of this figure is available in the online journal.)

PSFs were determined, each was subsequently subtracted from their corresponding $\mathrm{Z} \mathrm{CMa} \mathrm{component} \mathrm{to} \mathrm{determine} \mathrm{the} \mathrm{post-}$ subtraction residual flux. This residual flux is the dominant term in the calculation of the uncertainties in the $J$ - and $H$-band spectra.

To avoid saturation on such a bright target, the outburst data obtained with NIRC2 at Keck Observatory on UT 2008 December 21 utilized the $J_{\text {cont }}, H_{\text {cont }}, K_{\text {cont }}$, and polycyclic aromatic hydrocarbon narrow filters with central wavelengths $1.213 \mu \mathrm{m}, 1.580 \mu \mathrm{m}, 2.271 \mu \mathrm{m}$, and $3.290 \mu \mathrm{m}$, respectively. The flux values in these narrower filters were then scaled to corresponding broadband $J H K L$ wavelengths using the ratio of bandpasses between the two sets. The Z CMa system was observed using three-point dithering, and airmass corrected using the calibration stars listed in Table 1.

The left panel of Figure 2 shows the photometry of each component in the quiescent state collected from Koresko et al. (1991) using speckle interferometric observations. The right panel of Figure 2 shows our $J$ - and $H$-band $R \sim 45$ spectra from Palomar, as well as our $H K L$-band photometry obtained at Keck for both members of the system taken from the 2008-2009 outburst observations.

\subsection{High-resolution K-band Spectroscopy During Quiescence}

Spatially resolved $K$-band spectra of the two Z CMa components were obtained with the NIRSPEC spectrograph mounted behind the AO system on the W. M. Keck II telescope (Wizinowich et al. 2000) on UT 2006 December 17. The $K$-band setup provided wavelength coverage across the $\mathrm{CO}$ band head, the $\mathrm{Br} \gamma$ line, an $\mathrm{He}$ I line, and an $\mathrm{Mg}$ I and $\mathrm{Al}$ I metallic line region, but the spectral coverage is not continuous. The $R=\lambda / \Delta \lambda \sim 25,000$ observations were obtained at two position angles: $60^{\circ}$ (along the jet axis) and $120^{\circ}$ (along the projected semimajor axis of the binary). The slit was 2 .'26 long and data were taken using two different widths: $00^{\prime} 068$ and $0{ }^{\prime} .027$. MCDS readout mode was used with $30 \mathrm{~s}$ and $60 \mathrm{~s}$ integration times with five co-adds. Because of the complexity of the field, in addition to dithered pairs of spectra taken in an ABBA nod pattern, offtarget sky images were also obtained. Telluric correction was achieved using the rapidly rotating early-type star S Mon and further calibration was derived from internal arc line lamps and flat-field lamps. Figure 3 shows the flux from two echelle orders from NIRSPEC, spanning 2.10-2.13 $\mu \mathrm{m}$ and 2.29-2.32 $\mu \mathrm{m}$.

\section{RESULTS}

\subsection{Confirmation of the Outbursting Component}

The quiescent flux measurements taken from Koresko et al. (1991) and shown in Figure 2 reveal that shortward of $\sim 2 \mu \mathrm{m}$, the overall system flux is dominated by the SE component (blue curve in Figure 2). Longward of $\sim 2 \mu \mathrm{m}$, the NW component (the Herbig $\mathrm{Ae} / \mathrm{Be}$ star shown by the red points) dominates. Further, the NW component has increased in brightness during outburst by an order of magnitude over the quiescent values. At the same time, we find a $\sim 30 \%$ flux decline between the quiescent and outburst states for the SE component (the FU Ori star), shown in blue. The high angular resolution afforded by the Keck and Palomar AO systems allows us to confirm the claim (Malbet et al. 2010; Benisty et al. 2010; Szeifert et al. 2010) that the NW component is responsible for the ensemble system's increase in brightness.

\subsection{Two-component Model Fits to Data}

Using the photometry obtained at Keck and Palomar, as well as the work of Koresko et al. (1991), we are able to satisfactorily fit two-component, reddened, blackbody models to the quiescent and outburst spectral energy distributions for each member of the Z CMa system shown in Figure 2. For the NW component, our model that is physically plausible for such a luminous star is made up of two blackbodies of temperatures 8500 and $1100 \mathrm{~K}$ behind $A_{V}=10$. For the SE member, we find a physical match using components of 5500 and $900 \mathrm{~K}$ behind $A_{V}=5.2$. The reddening uses an optical total-to-selective extinction ratio, $R_{v}=3.1$, and the Mathis (1990) extinction curve. Although we have chosen to refrain from assigning a great deal of physical meaning to this two-component model, we refer to these components as "photosphere" and "disk." In each of the quiescent and outburst phases, the disk blackbody temperatures, normalizations, and reddening values are identical. At the same time, the normalization of the fit to the photospheric component 


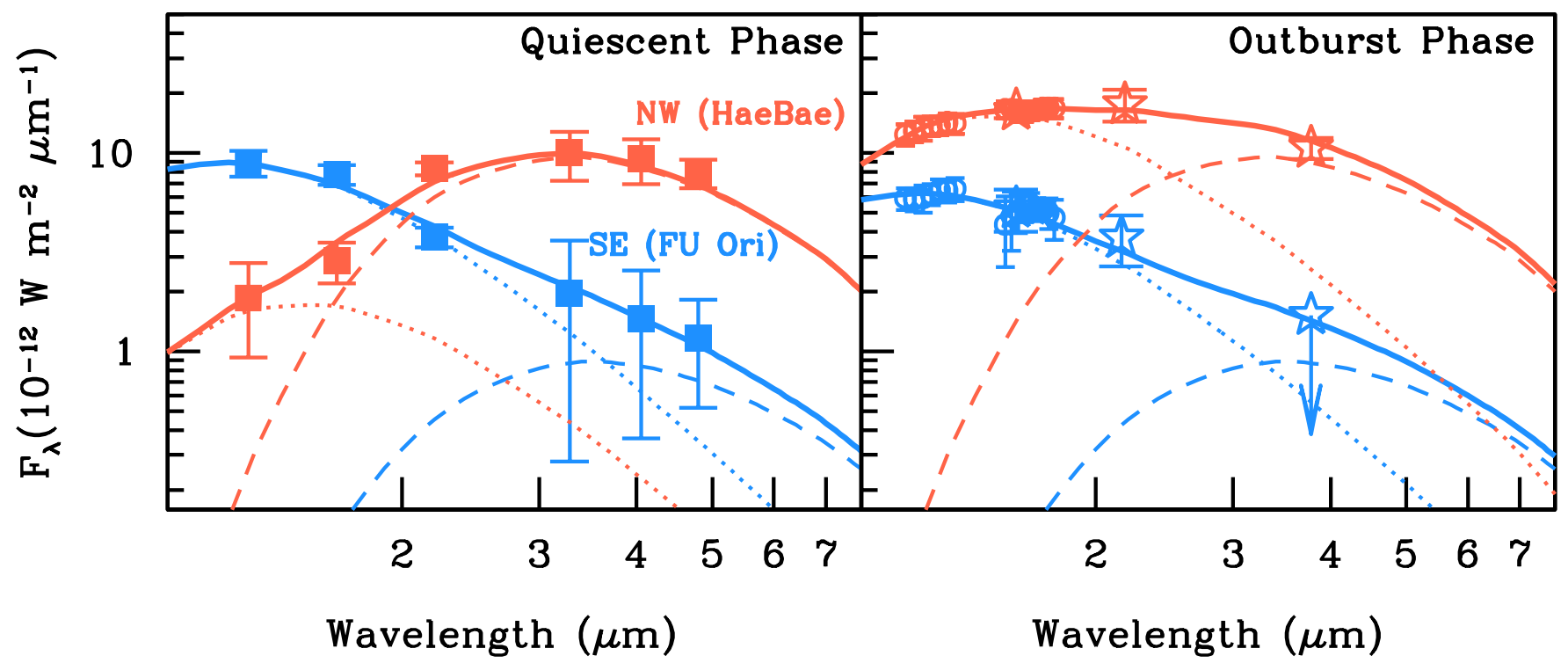

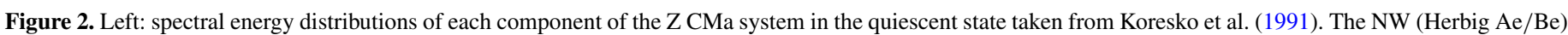

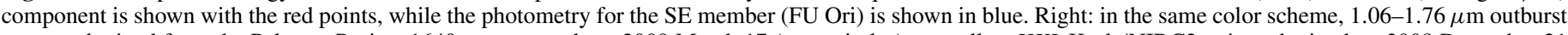

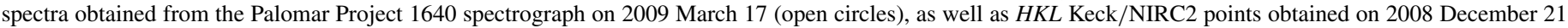

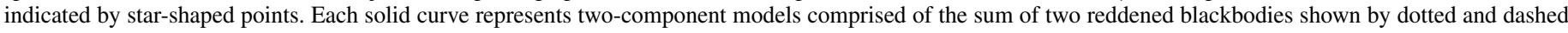
lines. The 2008 outburst of this system is conclusively due to the order-of-magnitude brightness increase by the Herbig Ae/Be component.

(A color version of this figure is available in the online journal.)

of the NW member has increased by an order of magnitude from quiescence to outburst, while for the SE component, it has been reduced by $30 \%$ between the two stages. The inherent variability of the $\mathrm{Z} \mathrm{CMa} \mathrm{system} \mathrm{is} \mathrm{reflected} \mathrm{in} \mathrm{these} \mathrm{changing} \mathrm{photosphere}$ normalizations.

Figure 2 shows that in addition to the order-of-magnitude brightness increase of the NW component, the peak luminosity of this member has shifted blueward, indicating either a greater luminosity of the photospheric component or a significant decrease in the $A_{V}$ value. However, decreasing the $A_{V}$ value immediately requires unphysical values for the photospheric temperature. Specifically, $A_{V}=2$ for the NW component implies an unphysical best-fit photosphere temperature of $\sim 1300 \mathrm{~K}$. The $5500 \mathrm{~K}$ photospheric temperature for the SE member agrees well with typical FU Ori objects that show $\mathrm{CO}$ absorption features (See Section 3.3) as described, for example, in Calvet et al. (1991). Meanwhile, the disk temperature for the NW member agrees well with values quoted in Szeifert et al. (2010). Lastly, this $\sim 30 \%$ decrease in flux for the SE component over $\sim 20$ years is remarkably similar to the optical decay reported in Clarke et al. (2005) for the prototypical system FU Ori.

\subsection{CO Features Observed in Quiescence}

The NIRSPEC spectra shown in Figure 3 capture the prominent $\mathrm{He}$ I emission line at $2.113 \mu \mathrm{m}$ as well as the $\mathrm{CO}$ band head at $2.294 \mu \mathrm{m}$. CO emission in this wavelength range occurs in $\sim 20 \%$ of luminous young stellar systems (e.g., Carr 1989), and probes the innermost regions of the circumstellar accretion disks (Najita et al. 2008).

Our Keck high angular resolution observations allow us to determine that the SE component (the FU Ori star) possesses a significant CO band head absorption at $2.294 \mu \mathrm{m}$. Several FU Ori objects such as FU Ori itself, V1057 Cyg, and V1515 Cyg show significant absorption features due to this $\mathrm{CO}$ band head (e.g., Reipurth \& Aspin 1997). It has been noted that Z CMa possesses rather weak $\mathrm{CO}$ absorption at $2.294 \mu \mathrm{m}$ relative to

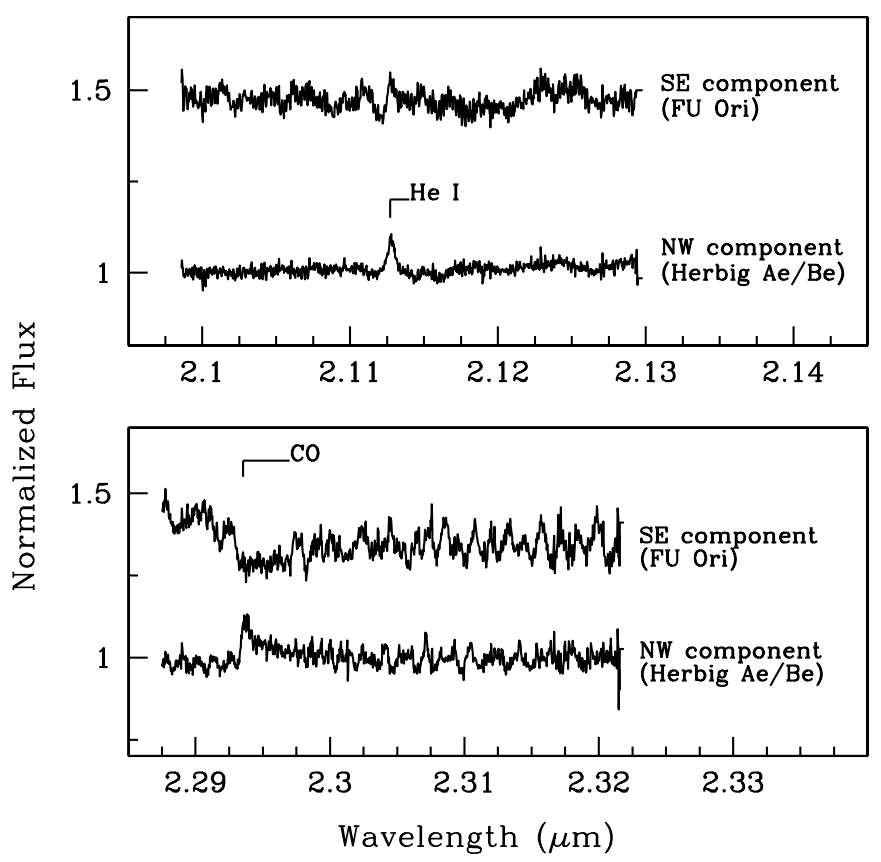

Figure 3. Keck $K$-band spectra from the NIRSPEC spectrograph for both the $\mathrm{SE}$ and NW components of Z CMa obtained while the system was in quiescence on 2006 December 17. The Herbig Ae/Be component shows a prominent CO emission feature located at $2.293 \mu \mathrm{m}$, while the FU Ori component shows a significant absorption at this wavelength.

other FU Ori stars, attributed to mixing of the signals between the two components due to inadequate angular resolution (Hartmann \& Kenyon 1996). We can check this hypothesis with our high angular resolution observations. Our clear detection of the absorption band head at $2.294 \mu \mathrm{m}$ in the SE component confirms the claim of van den Ancker et al. (2004) that the absorption is most likely arising from this component. While Liljestroem \& Olofsson (1997) present evidence for the CO band 
head using medium resolution $K$-band spectra (see especially their Figure 1), the spectral resolution of their observations was not sufficient to ascribe these $\mathrm{CO}$ band head features to one component or the other. Indeed, these authors attempt a decomposition of the spectrum by choosing a linear analytic function for the NW component, which forces deviations from this linear form to be due to the SE component.

At the same time, Figure 3 shows that the NW companion (the Herbig $\mathrm{Ae} / \mathrm{Be}$ ) shows an emission feature at the band head location. Malbet et al. (2010) and Benisty et al. (2010) note the presence of this $\mathrm{CO}$ overtone line in emission during outburst in their spatially unresolved observations of the Z CMa system. Our high spectral resolution observations clearly demonstrate the $\mathrm{CO}$ emission arises from the NW component. Calvet et al. (1991) discuss at length the conditions under which an object may exhibit $\mathrm{CO}$ emission during an outburst phase through the increased irradiation of the atmosphere of the circumstellar disk.

\section{CONCLUSIONS}

We present high angular resolution near-infrared observations of the Z Canis Majoris system during its 2008 outburst as well as prior to this during a quiescent phase. The $J H K L$-band outburst photometry conclusively determines (1) that the brightening is due solely to the embedded Herbig Ae/Be member, confirming results from earlier works, and (2) that the optically visible FU Ori component has actually experienced slightly declining brightness between the quiescent and outburst stages. Thus, this substantial brightness increase of the Herbig Ae/Be component relative to its quiescent state is responsible for the continuum brightness doubling of the ensemble system. Further, the Keck high-resolution $K$-band spectra obtained during a quiescent phase allow us to conclusively determine that the $2.294 \mu \mathrm{m}$ $\mathrm{CO}$ absorption feature seen in composite spectra of the system is due solely to the FU Ori component. In addition, however, these $K$-band observations show a prominent $\mathrm{CO}$ emission feature in the Herbig $\mathrm{Ae} / \mathrm{Be}$ member which likely dilutes the strength of the corresponding absorption feature in the SE component when the images of each component are blended with lower angular resolution observations. This result provides clarification of the source of the $\mathrm{CO}$ emission presented in other works (e.g., Benisty et al. 2010; Malbet et al. 2010).

This work was performed in part under contract with the California Institute of Technology (Caltech) funded by NASA through the Sagan Fellowship Program. A portion of this work was supported by the National Science Foundation under grant Nos. AST-1203023, AST-0804417, 0334916, 0215793, 0520822, and 1245018. A portion of the research in this Letter was carried out at the Jet Propulsion Laboratory, California Institute of Technology, under a contract with the National Aeronautics and Space Administration, and was funded by internal Research and Technology Development funds. The authors thank Pat Hartigan for his help obtaining the Keck/NIRSPEC observations. Some of the data presented herein were obtained at the W. M. Keck Observatory, which is operated as a scientific partnership among the California Institute of Technology, the University of California, and NASA. The Observatory was made possible by the generous financial support of the W. M. Keck Foundation.

\section{REFERENCES}

Antoniucci, S., Arkharov, A. A., Giannini, T., et al. 2009, ATel, 2024, 1

Benisty, M., Malbet, F., Dougados, C., et al. 2010, A\&A, 517, L3

Bonnell, I., \& Bastien, P. 1992, ApJL, 401, 31

Calvet, N., Patino, A., Magris, G. C., \& D’Alessio, P. 1991, ApJ, 380, 617

Canovas, H., Min, M., Jeffers, S. V., Rodenhuis, M., \& Keller, C. U. 2012, A\&A, 543, A70

Carr, J. S. 1989, ApJ, 345, 522

Clariá, J. J. 1974, A\&A, 37, 229

Clarke, C., Lodato, G., Melnikov, S. Y., \& Ibrahimov, M. A. 2005, MNRAS, 361,942

Covino, E., Terranegra, L., Vittone, A. A., \& Russo, G. 1984, AJ, 89, 1868

Crepp, J. R., Pueyo, L., Brenner, D., et al. 2011, ApJ, 729, 132

Dekany, R. G., Brack, G., Palmer, D., et al. 1998, Proc. SPIE, 3353, 56

Grankin, K. N., \& Artemenko, S. A. 2009, IBVS, 5905, 1

Hartmann, L., \& Kenyon, S. J. 1996, ARA\&A, 34, 207

Hartmann, L., Kenyon, S. J., Hewett, R., et al. 1989, ApJ, 338, 1001

Herbig, G. H. 1960, ApJS, 4, 337

Herbst, W., Racine, R., \& Warner, J. W. 1978, ApJ, 223, 471

Hinkley, S., Carpenter, J. M., Ireland, M. J., \& Kraus, A. L. 2011a, ApJL, 730,21

Hinkley, S., Monnier, J. D., Oppenheimer, B. R., et al. 2011b, ApJ, 726, 104

Hinkley, S., Oppenheimer, B. R., Brenner, D., et al. 2010, ApJ, 712, 421

Hinkley, S., Oppenheimer, B. R., Soummer, R., et al. 2009, ApJ, 701, 804

Hinkley, S., Oppenheimer, B. R., Soummer, R., et al. 2007, ApJ, 654, 633

Hinkley, S., Oppenheimer, B. R., Zimmerman, N., et al. 2011c, PASP, 123,74

Koresko, C. D., Beckwith, S. V. W., Ghez, A. M., Matthews, K., \& Neugebauer, G. 1991, AJ, 102, 2073

Koresko, C. D., Beckwith, S. V. W., \& Sargent, A. I. 1989, AJ, 98, 1394

Kraus, A. L., \& Ireland, M. J. 2012, ApJ, 745, 5

Leinert, C., \& Haas, M. 1987, A\&A, 182, L47

Liljestroem, T., \& Olofsson, G. 1997, ApJ, 478, 381

Malbet, F., Benisty, M., Dougados, C., et al. 2010, Proc. SPIE, 7734, 773427

Mathis, J. S. 1990, ARA\&A, 28, 37

Millan-Gabet, R., \& Monnier, J. D. 2002, ApJL, 580, 167

Najita, J. R., Crockett, N., \& Carr, J. S. 2008, ApJ, 687, 1168

Oppenheimer, B. R., Beichman, C., Brenner, D., et al. 2012, Proc. SPIE, 8447, 844720

Oppenheimer, B. R., Brenner, D., Hinkley, S., et al. 2008, ApJ, 679, 1574

Oppenheimer, B. R., \& Hinkley, S. 2009, ARA\&A, 47, 253

Poetzel, R., Mundt, R., \& Ray, T. P. 1989, A\&A, 224, L13

Pueyo, L., Crepp, J. R., Vasisht, G., et al. 2012a, ApJS, 199, 6

Pueyo, L., Hillenbrand, L., Vasisht, G., et al. 2012b, ApJ, 757, 57

Reipurth, B., \& Aspin, C. 1997, AJ, 114, 2700

Reipurth, B., \& Aspin, C. 2004, ApJL, 608, 65

Roberts, L. C., Jr., Rice, E. L., Beichman, C. A., et al. 2012, AJ, 144, 14

Sivaramakrishnan, A., Koresko, C. D., Makidon, R. B., Berkefeld, T., \& Kuchner, M. J. 2001, ApJ, 552, 397

Soummer, R. 2005, ApJL, 618, 161

Szeifert, T., Hubrig, S., Schöller, M., et al. 2010, A\&A, 509, L7

Thiebaut, E., Bouvier, J., Blazit, A., et al. 1995, A\&A, 303, 795

van den Ancker, M. E., Blondel, P. F. C., Tjin A Djie, H. R. E., et al. 2004, MNRAS, 349, 1516

Wang, H., Apai, D., Henning, T., \& Pascucci, I. 2004, ApJL, 601, 83

Whitney, B. A., Clayton, G. C., Schulte-Ladbeck, R. E., et al. 1993, ApJ, 417,687

Wizinowich, P., Acton, D. S., Shelton, C., et al. 2000, PASP, 112, 315

Zimmerman, N., Oppenheimer, B. R., Hinkley, S., et al. 2010, ApJ, 709, 733 